\title{
Erythrocytes 3D genome organization in vertebrates
}

\author{
Taskina A. ${ }^{1,2 *}$, Ryzhkova A. ${ }^{2}$, Khabarova A. ${ }^{2}$, Fishman V. ${ }^{1,2}$, Battulin N. ${ }^{1,2}$ \\ ${ }^{1}$ Novosibirsk State University, Novosibirsk, Russia \\ ${ }^{2}$ Institute of Cytology and Genetics SB RAS, Novosibirsk, Russia \\ *e-mail: a.taskina@g.nsu.ru
}

Key words: 3-dimensional nuclear organisation, Hi-C, erythrocytes, vertebrates

Motivation and Aim: 3-dimensional organization is essential for genome folding and compactization. It has been shown that progression through mitosis is accompanied by spatial rearrangement of the chromatin. This process could be studied using Hi-C technique, which shows genome-wide gain of spatial interaction between loci located $\sim 10 \mathrm{Mb}$ away from each other during mitotic prophase [1]. Previously we found that the same feature of spatial organization is also present in Hi-C maps of chicken erythrocytes that are in G2 phase [2]. Mechanism of formation and conservativeness in evolution of this phenomenon remain unknown. To resolve this issue, we investigated Hi-C data of blood cells in different classes of vertebrates. Methods and Algorithms: We used publically available erythrocyte Hi-C data of blood samples from fugu and catfish (bony fish), toads (amphibians), monitor lizard and sea turtle (reptilians), chicken and cassowary (birds). For human and mouse, nucleated terminal differentiation erythroid cells were used (unpublished data provided by R.A and A.A). Hi-C contacts were obtained using Juicer and processed with cooler software. We selected scaffolds longer than $9 \mathrm{Mb}$ and computed the contact frequency $(\mathrm{P})$ as a function of genomic distance (s), the $\mathrm{P}(\mathrm{s})$ function using cooltools expected function.

Results: The mitotic-like long-range interactions are clearly visible on Hi-C maps of amphibians, reptilians, mouse and bird. In accord with this, we observed the local peak of $\mathrm{P}(\mathrm{s})$ curve for these data, as well as for mammalian samples. Position of the peak varies from $20 \mathrm{Mb}$ in birds to $30 \mathrm{Mb}$ in amphibians. We didn't find the presence of the the peak in fish. Also, using chicken data we found that the local peak of $\mathrm{P}(\mathrm{s})$ curve is present only in the first five and the $\mathrm{Z}$ chromosomes, which are larger than $40 \mathrm{Mb}$. Position of the peak is constant in these six chromosomes.

Conclusion: Constant position of the peak in different long chromosomes data suggests a general mechanism explaining formation of the long-range mitotic-like interactions. Whether short chromosomes are packed in a different manner, or current method can not resolve the peak due to smaller number of faraway loci remains to be answered. The mitotic-like feature presents in erythrocytes among each considered class, besides bony fish. This could be explained by smaller size of bony fish chromosomes (less than $40 \mathrm{Mb}$ ).

Acknowledgements: This work was supported by RFBR grant \# 18-04-00668 A.

\section{References}

1. Gibcus JH, Samejima K, Goloborodko A et al. A pathway for mitotic chromosome formation. Science. 2018;359(6376):eaao6135. doi:10.1126/science.aao6135

2. Fishman V, Battulin N, Nuriddinov M et al. 3D organization of chicken genome demonstrates evolutionary conservation of topologically associated domains and highlights unique architecture of erythrocytes' chromatin. Nucleic Acids Res. 2019;47(2):648-665. doi:10.1093/nar/gky1103 